\title{
L'amélioration du cheptel cambodgien
}

\author{
par M. BARADAT
}

Une comparaison de l'effectif du cheptel cambodgien avec celui des pays voisins (Cf Tableau) ne permet cependant pas de regarder avec complaisance les méthodes d'élevage utilisées. Certes, avec 10 bovins (bcuf et buffle) au kilomètre carré et 1 bovin pour 3 habitants, le Cambodge occupe une situation privilégiée dans l'ensemble de la Fédération Indochinoise où l'on compte $l$ bovin pour 7 habitants et 6 têtes au kilomètre carré.

Ce n'est pas tellement l'importance numérique qui assure la valeur du cheptel mais tout autant la qualité individuelle et le rendement des sujets qui composent l'effectif. Si on le met en parallèle avec celui de pays tempérés, le cheptel cambodgien n'est guère favorisé. Vivant sur des terres généralement pauvres, à maigre végétation, soumis à des variations alimentaires très rudes par le jeu des saisons et des moussons alternées, le bétail du Cambodge est de faible valeur et médiocre apparence.

Un accroissement numérique qui ne peut dépasser le double de l'effectif actuel peut être obtenu, en une dizaine d'années, par l'amélioration des méthodes d'élevage, la transformation de l'éleveur et l'amélioration du milieu. Le rendement de l'exportation tient à l'utilisation des terres d'embouche et à l'excellence de ses herbages, la qualité du pâturage conditionnant étroitement la qualité du cheptel.

Il serait trop long. et en dehors du cadre de cette étude, d'examiner les possibilités d'amélioration du pâturage naturel, d'introduction des graminées exotiques, d'irrigation et fertilisation des prairies, de conservation de fourrages .. et de préciser quelles réalisations pourraient être immédiatement recherchées. Il suffit que l'on soit bien convaincu de la vérité de cet aphorisme " le bétail se fait par la bouche » et de ce que l'amélioration du bétail n'est possible qu'avec une pâture abondante et de bonne qualité.

De meilleures conditions d'entretien ne modifient pas définitivement les formes et les aptitudes du bétail. Mieux nourris, surtout à l'époque de la croissance, les bœufs et les buffles gagnent de la taille, du poids, leurs formes s'épanouissent. Mais ces modifications corporelles sont éphémères, elles ne dépassent pas l'individu qui les porte et les causes qui les ont fait naitre; elles ne sont pas héréditaires. Ainsi, on peut trouver commode de prendre en élevage des berges des taureaux destinés à la reproduction dans un milieu moins avantagé. Non seulement ces taureaux s'accommodent mal des nouvelles pâtures et leur fécondité s'en ressent, mais aussi leur descendance ne trahit aucun souvenir du format amplifié, apanage du bétail des berges. Tout au plus, on observe une amélioration de la précocité lorsque le taureau est issu d'une bonne lignée, avec un patrimoine héréditaire solide.

Comparaison du cheptel au Cambodge et dans les pays voisins

\begin{tabular}{|c|c|c|c|}
\hline & BCEUFS & BUFFLES & TOTAL \\
\hline & & ! & \\
\hline Cambodge ........ & 915.000 & 320.000 & 1.235 .000 \\
\hline Laos $\ldots \ldots \ldots \ldots \ldots \ldots$ & 350.000 & 400.000 & 750.000 \\
\hline Cochinchine......... & 262.600 & 277.500 & 540.000 \\
\hline Annam ....... & 630.000 & 320.000 & 950.000 \\
\hline Tonkin . & 170.000 & 410.000 & 580.000 \\
\hline Siam (1921) $\ldots \ldots \ldots \ldots$ & 4.410 .000 & 4.570 .000 & 8.980 .000 \\
\hline Indes et Birmanie.... & 160.000 .000 & 40.000 .000 & 190.000 .000 \\
\hline Phillippines . . . . . . . . & 1.021 .169 & 1.824 .842 & 2.846 .011 \\
\hline Madagascar (1921).... & 7.830 .000 & & 7.830 .000 \\
\hline
\end{tabular}

La force conservatrice est l'hérédité et l'éleveur ne peut espérer un perfectionnement animal durable que s'il prend grand soin de choisir les reproducteurs. Le mâle ou la femelle participent également à la formation de la descendance, mais un même mâle pouvant féconder plusieurs femelles, les mâles ont une influence dominante sur l'élevage en raison de leurs nombreux produits.

L'éleveur cambodgien ne cherche généralement pas à influencer la descendance et laisse la reproduction se faire au hasard. Souvent, les meilleurs mâles sont castrés de bonne heure pour être transformés en animaux de trait et les moins heureusement conformés, dont on ne peut espérer un profit immédiat, sont abandonnés à l'œuvre de reproduction; c'est de la sélection à rebours.

Les aptitudes qu'il convient de développer, d'améliorer et d'épurer sur le bétail cambodgien sont de deux sortes : une meilleure adaptation au travail, puisque boufs et buffles sont avant tout des animaux tracteurs, et une meilleure adaptation pour 
la production de la viande puisque l'aboutissement normal de ces animaux, des bœufs surtout, est la boucherie. Cette amélioration est possible, soit par sélection, soit par croisement. Je n'examinerai pas l'amélioration de l'aptitude laitière qui est inexistante chez les femelles de race autochtone. La production laitière se place au sommet de I'industrie pastorale par une transformation plus poussée des denrées fourragères en produits assimilables par l'homme; mais son incompatibilité avec les conditions d'élevage et de climat, avec la mentalité des paysans interdit de lui réserver une place particulière dans l'élevage cambodgien.

\section{SÉLECTION}

La sélection repose sur le choix des meilleurs reproducteurs. On doit castrer les mâles défectueux. Lorsque la sélection est continuée sur plusieurs générations, le' perfectionnement des aptitudes s'accentue à mesure. Au cours de l'amélioration ainsi conduite, les races de bœufs ou de buffles conservent toutes leurs qualités d'aclaptation au milieu, de résistance au climat. Les reproducteurs d'élite sont d'abord une minorité, mais deviennent une majorité qui s'accroit sans cesse lorsque des éleveurs nombreux unissent leurs efforts dans ce sens et s'associent dans une organisation méthodique.

Pour conserver et épurer les qualités du bétail cambodgien, il importe de les bien connaître, et les éleveurs appuieront leur choix sur les modes traditionnels d'appréciation., Ce bétail est rustique, ardent, vigoureux malgré sa petite taille, adapté aux difficultés saisonnières, au climat et assez résistant aux maladies tropicales. L'amélioration vise à l'amplifier, à lui donner plus de taille, plus de volume, c'est-à-dire plus de,puissance au démarrage et à la traction, tout en lui conservant son endurance et sa vivacité.

Les taureaux seront choisis parmi les jeunes mâles vigoureux, râblés à formes saines, ayant un développement corporel au-dessus de la moyenne. On recherchera une encolure vigoureuse et masculine; les membres antérieurs bien écartés; une poitrine profonde, ample; des côtes longues, bien voussées, remontant loin en arrière; un dos droit et large; un rein ample et musclé; une croupe horizontale, large, avec une fesse rebondie et bien descendue. Les membres seront compacts, bien d'aplomb, les articulations solides, bien développées. En bref, souplesse et vigueur sont les qualités dominantes qui doivent etre recherchées.

Ces mêmes qualités corporelles doivent être recherchées sur les buffles étalons. Cet animal travaillant plus par sa masse que par son énergie, on cherchera à se rapprocher d'un type massif, près de terre, à côtes serrées, sans abdomen exagéré, avec des membres robiustes, des articulations larges.

Les buffles du Cambodge présentent deux variétés assez tranchées dans des conditions d'habitat très affirmées et aussi toutes les gammes intermédiaires. Les éleveurs distinguent la variété Krabey beng ou buffle des marais et la variété Krabey loeu ou buffle des terres hautes. Le premier type est plus massif, plus éclalé, plus près de lerre, avec des proportions moins heurtées et un aspect de grande puissance. Il ne peut cependant être transféré en des régions où il ne retrouverait pas une alimentation identique, copieuse et riche en sucs. Le choix des taureaux doit se faire dans les élevages du cru. L'acclimatement du Krabey loeu est possible partout. On peut rapprocher ces différences de comportement - et les Cambodgiens avisés ne manquent pas de le faire de celles qu'on observe entre deux variétés d'éléphants domestiques dont l'habitat est superposable, l'éléphant des terres inondées, damrey ronéam et l'éléphant des collines, damrey phnom. Comme le buffle des terres hautes, l'éléphant des montagnes est, à juste titre, prisé davantage.

Le buffle ne peut $s$ 'améliorer que par sélection. Il paraît inutile d'envisager au Cambodge le croisement avoc le buffle de l'Inde qui, hormis une aptitude laitière développée, n'offre aucune supériorité dans l'aptitude motrice. Les importations de buffles de l'Inde au Tonkin furent un échec par suite d'une indifférence génitale avec la race autochtone. On doit noter, cependant, que ce croisement a donné de bons résultats entre les mains des éleveurs philippins.

Une bonne fécondation peut être assurée par l'emploi d'un taureau pour une quarantaine de femelles.. Néanmoins, dans les troupeaux entretenus sur terres sablonneuses et latéritiques où la sécheresse dépouille le pâturage, les chaleurs sont de si courte durée et si peu marquées que la proportion des taureaux doit être accrue.

De même dans les troupeaux de bufflesses, où il est sage de prévoir 1 taureau pour 25 femelles.

La conservation de la plupart des femelles étant encore indispensable pour l'accroissement numérique du cheptel, seules les plus mal conformées seront éliminées. La sélection ne sera cependant pas unilatérale. Les qualités de précocité, de fécondité, l'aptitude à nourrir les produits seront recherchées et les femelles qui les affirment au plus haut degró doivent être conservées jusqu'à épuisement de leurs facultés reproductrices.

Outre l'appréciation des caractères plastiques, les concours locaux d'élevage sont orientés vers une recherche du rendement qui est seule susceptible d'apporter quelque méthode dans la sélection. L'appréciation des mâles selon leur conformation 
mérite d'être étayée par un contrôle des performances (épreuves de traction lourde, d'endurance au labour, de traction routière à allure rapide). Dans les élevages qui se livrent à la production du bœuf de boucherie, les concours d'animaux abattus doivent completer les enseignements des concours d'animaux gras et permettre de diriger les éleveurs.

Le choix des taureaux devra, si possible, s'accompagner d'une étude de l'ascendance. Quand les mêmes qualités corporelles, les mêmes aptitudes existent chez les parents immédiats et, si l'on peut remonter au-delà, chez les ancêtros de l'animal, il s'y trouve une garantie qu'elles sont héréditaires et ont chance d'être léguées aux futurs produits. Lorsqu'un taureau est issu d'une famille à ressemblance homogène, on peut l'espérer pourvu d'une puissance héréditaire irrésistible qui le rend bon raceur.

L'examen de la descendance éclaire l'œuvre de sélection et révèle le géniteur de qualité mieux que l'appréciation isolée de l'animal. Il va de soi que les taureaux pourvus d'une grande force de transmission personnelle doivent être conservés en fonction autant que cela est possible.

On ne peut cependant fonder de grands espoirs sur l'entreprise de sélection du cheptel. Dans ce façonnage du bétail, il faudrait une collaboration active de l'éleveur durant plusieurs années, permanence d'effort contraire à la nature du paysan cambodgien. Le choix des reproducteurs n'est pas favorisé par l'existence de types faciles à identifier selon leur plastique. Le bétail cambodgien présente la plus grande diversité et se trouve en état de variation désordonnée. Il est composé de sujets hétéroclites réunis par la trame d'une vague ressemblance. On est frappé des différences morphologiques séparant les sujets d'un même troupeau qui vivent cependant dans les mêmes conditions. Il n'existe aucun critère permettant d'affirmer qu'un reproducteur, magnifiquement doué du point de vue de la beauté plastique, transmettra ses qualités à sa descendance. Dans la lignée, les types aberrants, dissidents, seront d'autant plus nombreux que le patrimoine héréditaire est impur.

L'éleveur cambodgien n'a eu, jusqu'ici, qu'un maigre entendement de l'œuvre de sélection. Or, c'est un fait, les races domestiques, si on les veut productives au plus haut point, doivent être modelées par l'homme et non pas du tout par des influences tenant au milieu. L'ignorance des Cambodgiens en matière de sélection est illustrée par l'existence des buffles albinos. L'albinisme n'est pas particulièrement prisé, au contraire, car les sujets atteints de cette décoloration sont plus sensibles à l'ardeur solaire, plus vite essoufflés au travail : " ils ne peuvent se mettre à l'ombre de leur peau ". Le nombre des albinos est tel que les superstitions qui vont aux êtres d'exception seraient, à leur égard, déplacées ; on ne saurait les rapprocher de l'éléphant blanc, autre albinos très rare, qui, lui, est divinisé. Or, l'albinisme est inconnu chez le buffle sauvage issu de la même souche que le buffle domestique, la sélection naturelle éliminant cette dissonance. L'albinisme étant, au surplus, un caractère dominant, il serait très facile aux éleveurs de s'en débarrasser avec quelque patience. Mais la continuité d'action est absente du comportement ordinaire des éleveurs cambodgiens. On ne saurait dresser un parallèle entre leurs conceptions d'une génétique animale et celles, moins sommaires, de la génétique végétale. Encore, s'agit-il là de connaissances liées à un esprit aiguisé d'observation. Ainsi, à Battambang, nous avons vu les paysans différencier avec virtuosité des espèces différentes de paddy, espèces multiples créées par mutations et hybridations naturelles, qui ne devaient presque rien à l'intervention des cultivateurs.

Il y a, certes, dans le bétail cambodgien, des qualités natives qui méritent d'être conservées, développées, épurées : rusticité, endurance, tempérament vif et nerveux et une certaine capacité d'assimilation qui le fait engraisser facilement avec des conditions favorables.

Il importerait de séparer dans ce bétail disparate un certain nombre de types, trois ou quatre, pas davantage, pour arriver à la création de races homogènes différentes les unes des autres par plusieurs caractères. C'est là une grande ceuvre irréalisable en milieu indigène. D'abord, comment tracer le portrait exact, définir le modèle typique susceptible de plaire aux ćlcveurs d'une même région? Et ensuite, comment être assuré qu'ils feront un effort pour ne pas s'écarter du standard soigneusement établi?

A partir du groupe ethnique actuel, si l'on excepte croisement et métissage, la création de races homogènes ne peut se concevoir que selon deux modes dont le second n'exclut pas nécessairement le premier :

$1^{\circ}$ Apparition de " raceurs 》 dont les particularités originales, les attributs de famille seront apparents, visibles chez tous ses descendants et seront mathématiquement légués; événement problématique et que seul un éleveur averti peut découvrir à temps ;

$2^{\circ}$ Euvre de sélection où l'éleveur trie les attributs importants, élimine les divergences, écartant à mesure des hérédités qui se combattent.

\section{CROISEMENT}

Il serait vain d'attendre de la seule sćıłection, un perfectionnement du bétail autochtone allant de pair avec l'amélioration rapide du sol et des 
herbages que l'on observe.sous l'extension des cultures des berges, le façonnage régulier des « chamkar» et les apports limoneux des "prek» et ouvrages de colmatage: La sélection ne peut faire apparaître du nouveau et des caractères inexistants dans la race cambodgienne.

Avec des moyens économiques et des résultats rapides, le croisement permet l'introduction de propriétés nouvelles.

Les zébus de l'Inde (races de Sind, d'Ongole) " ont été reconnus dans toute l'Indochine comme possédant un ensemble de qualités qui los désignont pour l'amélioration des bovins du pays en vue de la boucherie, du travail ou de la production laitière » (Jacotot, L'élevage en Indochine, Revue "Indochine $\gg ; 5$ novembre 1942).

Amplification du format et de la masse contribuant à la puissance dynamique, résistance aux parasites sanguicoles, à la peste bovine, rusticité et endurance sont les principaux avantages à inscrire en faveur de ces croisements. Les métis de première génération réunissent les qualités des deux races et; en milieu convenable, forment des sujets réussis. On peut leur reprocher de n'être pas plus précoces que la race autochtone, d'avoir des allures moins vives, tout en demeurant aussi ombrageux. En boucherie, il y a seulement augmentation du format, mais le rendement n'est pas accru, non plus que la finesse de chair et la tendance à l'engraissement. Ces défauts, qui sont peu marqués dans le croisement Sind, sont accusés sur le demi-sang Ongole, chez qui on relève une grande ampleur du train antérieur, le rapport entre le poids des quartiers de devant et celui des quartiers de derrière étant franchement supérieur à 1 .

Tels quels, ces croisements répondent au vœu le plus général de l'éleveur cambodgien qui reste une amelioration de la fonction travail. Les privileges physiologiques de ces zébus en font les seuls sujets susceptibles de perfectionner le bétail cambodgien dans les conditions actuelles de son entretien. Encore que ce croisement ne doive être conseillé que dans les conditions les plus favorables du milieu. Il serait vair de tenter l'élevage des métis de Sind ou d'Ongole sur savanes ou maigres rizières. Quoique peu exigeants; les métis ne peuvent s'accommoder d'une chiche pâture. Une insuffisance alimentaire durant la croissance aboutit à un défaut d'équilibre et à une médiocre fusion des formes. Le métissage $y$ est très instable et le retour à la race autochtone est très rapide, si bjen qu'en deux ou trois générations l'apport diı zébu de l'Inde n'est trahi que par' des particularités secondaires (profil céphalique, fanon, survivance de la robe grise...).

Par contre, avec une alimentation meilleure; sur pâturages des berges, le croisement Sind ou Ongole, surtout le premier, donne un amalgame harmonieux des caractères parentaux. Le métissage y provoque une amélioration instable avec quelques panachures et dissidences. Mais les méfaits du métissage, par dislocation et insurrection des caractères ancestraux, restent peu apparents, les sujets réussis étant les plus nombreux, 'selon une plastique et des proportions qui répondent au vœu général des éleveurs.

Il semble qu'il n'y ait pas lieu de rechercher, pour l'ensemble de l'élevage des berges, un croisement progressif, continu qui, en quelques générations, une trentaine d'années, conduirait à l'absorption complète et à la substitution à la race autochtone d'un métis reproduisant tous les caractères du zébu de I'Inde. Dans la majorité des cas, les limites opportunes du croisement s'arrêtent au demi-sang d'Ongole ou au trois quarts de sang du Sind. A ce stade, un croisement de retrempe, par utilisation d'un géniteur Sind avec des métis d'Ongole ou inversement, ne donne guère de mécomptes et les produits issus de ce façonnage, s'ils sont quelque peu disparates, répondent aux mêmes'valeurs économiques.

On peut se demander si le croisement avec les zébus de l'Inde ne doit pas être considéré comme un stade préparatoire, comme la production du porte-greffe indispensable à l'introduction de races plus nettement amélioratrices, selon ùne technique qui a fait ses preuves en Afrique du Nord, en Colombie et au Brésil. Au Cambodge, aucune expérimentation 'ne' permet de répondre à cette question par l'affirmative. Los rares sujets européens importés (de races bretonne pie noire, bordelaise, d'Abondance) furent utilisés sans discrimination, sans les correctifs essentiels des conditions de milieu et leur descendance s'est abâtardie très rapidement. On avait recherché une amélioration de la faculté laitière qui, à moindres frais, peut s'obtenir par l'infusion de sang zébu, si bien que ces expériences sont sans valeur concluante.

La possibilité de nourrir un veau exigeant est à la base de l'amélioration. Cette faculté est inexistante chez la vache cambodgienne; elle paraît suffisante chez la vache métisse du Sind ou d'Ongole. 'Si bien que, sur ce porte-greffe et dans les élevages les plus favorisés, le croisement serait possible avec des races réputées comme répondant aux valeurs recherchées par les paysans cambodgiens : la production du travail et. accessoirement, de la viande. Il est certain que la précocité, l'obtention d'une masse suffisante, d'une meilleure conformation pour la boucherie, d'une plus grande finesse de chair doivent être recherchées au-delà des zébus de l'Inde. L'expérimentation devra porter' sur l'infusion du sang limousin qui a affirmé sa rósistanco aux conditions sévères d'entretien' sur le' «'campo'» 
brésilien et sur l'utilisation du croisement Afrikander qui a fait ses preuves en Rhodésie, au Congo belge et aux Philippines dans des conditions mésologiques semblables à celles des pâtures de berge au Carnbodge. Ce n'est que lorsque l'épreuve en aura été faite sur plusieurs générations, qu'il pourra être opportun de sortir du cadre des stations zootechniques pour une mise en œuvre de ce croisement en milieu rural.

Pour quelques années encore, le seul croisement justifié demeure celui avec les races de l'Inde dont les affinités avec la race autochtone ne sont plus a démontrer.

Le programme actuel d'amélioration par croisement est sagement limité aux élevages de berge. En raison de variations dans la teneur en éléments minéraux, des alluvions du Mékong et des sols qui les reçoivent, le format du bétail va décroissant d'amont en aval. Cette particularité a servi à déterminer l'aire d'action attribuée à chaque groupe de géniteurs, le Sind étant destiné à l'amont et l'Ongole à la zone aval. Les défauts habituels aux zébus de l'Inde (manque de taille et allures réduites du Sind, manque d'ampleur et dysharmonie de l'Ongole) étant compensés par des valeurs opposées de l'élevage autochtone. Celte délimitation arbitraire offre également l'avantage d'apporter quelque méthode et de réduire les interférences des deux races utilisées pour $c \Theta$ croisement.

Ce programme a été mis en ceuvre avec des moyens réduits. Le nombre de taureaux améliorateurs est limité a la production des stations zootechniques. Il devra être renforcé par des importations de géniteurs d'élite acquis dans l'Inde qui permettront, en outre, de rafraîchir le sang.

Il va de soi qu'avec des méthodes d'élevage aussi peu perfectionnées que celles en usage, même sur paturages des berges, on ne peut mettre en œuvre que des formules simples. Celle en vigueur est la suivante : le nombre des femelles adultes ayant eté exactement recensé, dans chaque village, I taureau est placé à raison de 30 vaches. Tous les taurillons de race autochtone qui pourraient contrarier l'entreprise de croisement sont castrés périodiquement. De manière à former un bloc cohérent d'une même série ethnique, le croisement est étendu successivement aux villages voisins. L'émiettement, le balbutiement des entreprises de croisement isolées doit être proscrit. Pour créer une race nouvelle, il est indispensable de créer un groupe homogène suffisamment étendu. Le croisement avec un taureau de race pure (zébu de l'Inde ou zćbu autochtone) - croisement alternatif - devra être poursuivi aussi longtemps que possible pour éviter le métissage.

A la longue, aux frontières de ces groupes ethniques, s'observeront des éclaboussures du sang zébu indien qui seront rapidement absorbées par la race locale, le métissage n'ayant pas de chances de survivre dans un milieu hostile.

\section{UTILISATION DES TAUREAUX}

Le mode attardé de l'élevage au Cambodge contrarie l'entreprise d'amélioration du bétail. La ségrégation indispensable, l'élimination des sujets équivoques, défectueux, qui permet de cultiver les qualites et de supprimer les défauts d'une race supposent une collaboration active, permanente, méthodique de l'éleveur. Or, cette collaboration est inexistante et l'éducation du paysan ne peut être espérée de sitôt, qui permettrait de créer des associations efficaces pour tenir les livres généalogiques ou les livres d'élite ou même de créer des syndicats d'élevage dont tous les participants accepteraient les règles. Les disciplines qui sont à la base de l'amélioration rationnelle échappent à l'entendement commun. Alors que, pour l'homme, une eugénique élémentaire serait difficilement applicable, ce n'est pas dans la tradition bouddhique que l'on pourrait trouver les principes des contraintes qui président à la sélection telles que le choix des reproducteurs. On étonne beaucoup les Cambodgiens, qui ont accoutumé de laisser au hasard l'œuvre de reproduction, en leur affirmant que l'avenir de leur élevage dépend de la valeur des mâles. et des femelles.

Tant que, seul, l'ascendant du Service de l'Élevage pèsera sur les paysans pour les persuader, les retourner, les décider, l'amélioration du bétail sera ralentie des hésitations, de l'incompréhension et de l'entêtement de quelques-uns. Aucune orientation de l'élevage n'est possible si elle n'est régentée et si les pouvoirs publics ne contribuent pas à l'observance de contraintes qui ne peuvent être librement acceptées par ignorance des intéressés. Si la présente étude devait entraîner une collaboration efficace des autorités du Royaume, elle marquerait une étape et permettrait d'atteindre au but. Il est indispensable que des disciplines collectives soient imposées et que soit proscrite la liberté complète qui est encore de règle en matière d'élevage.

Le comportement des paysans, le défaut d'ascendant du Service de l'Élevage, l'indifférence des autorités provinciales ne sont pas les seuls principes d'achoppement. Bien des causes naturelles contrarient l'action des taureaux affectés d'une prime d'entretien ou d'une prime de conservation. Lors des concours itinérants, du contrôle des naissances, on s'aperçoit que le nombre des saillies, celui des produits sont réduits. L'activité des taureaux est gênée par celle des taurillons dignes de la castration, 
entravée par la multiplicité des élevages familiaux qui, en dispersant les troupeaux, rend aléatoire la rencontre des femelles en chaleur. La brièveté des chaleurs dans l'espèce zébu est un écueil majeur. Alors que chez les taurins façonnés par une longue domestication, les chaleurs sont bruyantes et durables, le comportement des femelles zébus reste beaucoup plus discret et les chaleurs durent rarement au-delà d'une journée. Pour détecter les femelles en chaleur et les atteindre en temps voulu, il faut que le taureau soit présent dans le troupeau. Cette condition réalisable en élevage extensif, l'est beaucoup moins en élevages familiaux où l'ensemble $\mathrm{du}$ troupeau du village n'est pas toujours réuni sur la même pâture. Il est curieux de constater que, sur les produits obtenus par croisement du zébu autochtone et du taureau d'Europe, le comportement des femelles durant l'ovulation reste calqué sur celui des vaches d'Europe. Les vaches métisses s'agitent et réclament bruyamment le mâle, comme si cet héritage d'une longue domestication était un caractère dominant. On le retrouve intact, en effet, après deux ou trois générations retournant à la race locale.

La fécondité des taureaux que l'on a choisis pour améliorateurs est donc limitée, sauf à l'époque ordinaire du rut, où l'ardeur des mâles, l'exaltation de l'instinct génésique des femelles les portent à vagabonder et à se rencontrer.

On peut done so demander si la pratique do l'insémination artificielle ne serait pas plus efficace, plus rapide et plus sûre et ne permettrait pas d'utiliser au maximum les taureaux réservés au croisement. Avec cette méthode, l'absorption de la race indigène par l'amélioration serait beaucoup plus rapide.

Bien que la collectivisation du troupeau ne soit pas chose faite au Cambodge, l'application de cette méthode doit être envisagée dès que possible. On y rencontrera cependant des obstacles sérieux: : l'indifférence des éleveurs, l'indocilité des vaches qui exigera une contention stricte. Le caractère furtif et secret des chaleurs embarrassera au plus haut point. Une action d'ensemble sera seule économique. Il sera nécessaire d'avoir en même temps sous la main plusieurs vaches en état réceptif, chez qui l'ovulation aura été artificiellement provoquée par injection de folliculine. Les propriétaires ne pouvant fournir aucun antécédent sur l'état génital de leurs vaches, pour éviter les risques d'avortement dû à une injection intempestive de folliculine, le troupeau aura été au préalable tenu a distance des taureaux et taurillons. L'opération pratiquée à la saison ordinaire du rut, après mise bas de la plupart des vaches, aura chance d'atteindre presque tout l'effectif. Un taureau sera, par la suite, placé dans le troupeau pour pallier les insuccès de l'opération. Au début de l'application de cette technique, les élevages des îles dụ Mékong, où la mise à l'écart des taureaux peut ctre facilement assurée, offriront les conditions les plus favorables de réussite. 